\title{
Modeling Neurodegeneration in Zebrafish
}

\author{
Yanwei Xi • Sandra Noble $\cdot$ Marc Ekker
}

Published online: 27 January 2011

(C) The Author(s) 2011. This article is published with open access at Springerlink.com

\begin{abstract}
The zebrafish, Danio rerio, has been established as an excellent vertebrate model for the study of developmental biology and gene function. It also has proven to be a valuable model to study human diseases. Here, we reviewed recent publications using zebrafish to study the pathology of human neurodegenerative diseases including Parkinson's, Huntington's, and Alzheimer's. These studies indicate that zebrafish genes and their human homologues have conserved functions with respect to the etiology of neurodegenerative diseases. The characteristics of the zebrafish and the experimental approaches to which it is amenable make this species a useful complement to other animal models for the study of pathologic mechanisms of neurodegenerative diseases and for the screening of compounds with therapeutic potential.
\end{abstract}

Keywords Zebrafish - Neurodegenerative diseases .

Parkinson's disease $\cdot$ Huntington's disease · Alzheimer's disease $\cdot$ Dopaminergic neuron

\section{Introduction}

The zebrafish has been widely used as a model for the study of developmental biology. One major reason for its

\author{
Y. Xi $\cdot$ S. Noble $\cdot$ M. Ekker $(\triangle)$ \\ Department of Biology, Center for Advanced Research \\ in Environmental Genomics, University of Ottawa, \\ 30 Marie Curie, \\ Ottawa, Ontario K1N 6N5, Canada \\ e-mail: mekker@uottawa.ca \\ Y. Xi \\ e-mail: yxi016@uottawa.ca \\ S. Noble \\ e-mail: noble.sandra@gmail.com
}

popularity is that not only is it a vertebrate and thus closer to humans than invertebrate models, but it has several advantages over other vertebrate models. These include high fecundity (a few hundred eggs per spawning), transparent embryos, and external development. Zebrafish can be easily visualized and experimentally manipulated. Their generation time is short (2-3 months). All these features, previously found mainly in invertebrate models, facilitate genetic and high-throughput functional studies.

The straightforward and yet powerful experimental approaches based on microinjection of antisense morpholino oligonucleotides (MOs) into one-cell stage embryos are commonly used to temporarily silence zebrafish genes during the first few days of embryonic development. MOs are chemically modified oligonucleotides that can specifically bind to their target mRNAs with more stability and resistance to endogenous degradations. Their hybridization to mRNA can block translation when they are targeted to sequences near the initiation codon. Alternatively, they can block splicing when targeted to exon-intron boundaries. This method has proven to be simple, rapid, specific, and effective $[1 \bullet \bullet$. Another widely used technique in zebrafish is transgenesis with a fluorescent reporter expressed under the control of a tissue- or cell-type-specific promoter. Transgenesis can also be used to overexpress specific genes or their mutated versions in specific tissues or cell types. The recent introduction of I-SceI meganuclease or of Tol2 transposon greatly increased the efficiency of transgenesis in zebrafish [2,3].

Zebrafish have also proven to be a particularly relevant vertebrate model for the study of human diseases. Compared with other vertebrate models, screens for zebrafish mutants are less costly and easier to perform. Large-scale mutagenesis screens have been performed in zebrafish [4]. Some of the mutants identified in these screens are 
reminiscent of human diseases and made great contributions to our understanding of human biology. Zebrafish mutants have allowed a better understanding of various human conditions including blood disorders, aging, muscular dystrophy, and autism. Here, we review recent progress in modeling human neurodegenerative disorders using zebrafish.

\section{Parkinson's Disease}

Although there are some notable differences in structure and scale between the zebrafish brain and that of humans, the overall organization shows similarities. Specific regions of the zebrafish brain can be related to and are often strikingly conserved when compared with their human counterparts. For example, the zebrafish ventral telencephalon is suggested to be homologous to the striatum in humans. Retrograde tracing experiments in adult zebrafish brain found that dopaminergic (DA) neurons projecting to the ventral telencephalon are located in the posterior tuberculum of the ventral diencephalon (vDC). Thus, zebrafish DA neuron clusters in the $\mathrm{vDC}$ are analogous to the ascending midbrain DA neurons of the mammalian nigrostriatal pathway [5]. The DA system has been characterized during embryogenesis in zebrafish. DA neurons are first detected at about 18 hours post-fertilization (hpf) in a cluster of cells in the vDC. At $72 \mathrm{hpf}$, the zebrafish central nervous system (CNS) development is well advanced. DA neurons are found mainly in the olfactory bulb, preoptic region, pretectum, retina, and vDC. Several mutants with abnormal patternings of DA neuron in the vDC have been identified in large-scale mutant screens. Characterization of these mutants suggest that the Shh and Nodal signaling pathways play key roles in DA neuron differentiation in zebrafish as they do in mammals. Transcription factors, including Nurr1/Nr4a2, Pitx3, and Lmx1b, have been shown to play evolutionarily conserved roles in the specification of zebrafish DA neurons.

To date, several orthologs of major Parkinson's disease (PD)-associated genes have been found in zebrafish, including parkin, pink1, dj-1, and lrrk2. MO knockdown and/or transgenic overexpression of mutants of these genes suggest that they have conserved functions in the development and/or survival of DA neurons.

Parkin

Mutations in Parkin are the most common autosomalrecessive mutations in early-onset PD. Parkin encodes an E3 ubiquitin ligase involved in the ubiquitin proteasome degradation system. Parkin is also thought to play critical roles in mitochondrial function together with other PD- related genes such as PINK1 and DJ-1. Drosophila models of parkin loss of function show a loss of DA neurons and reduced mitochondria with a remarkable loss of cristae [6, 7]. However, parkin-null mice do not develop any DA neuron loss or mitochondrial or behavioral abnormality [8]. The zebrafish parkin gene encodes a 458 amino acid protein, which is highly conserved between zebrafish and humans with overall $62 \%$ identity and up to $92 \%$ similarity in its functional domains [9]. Similar to human Parkin, zebrafish parkin is ubiquitously expressed throughout embryonic development and in adult tissues. MO knockdown of parkin resulted in an approximately $20 \%$ loss of DA neurons in the vDC with increased susceptibility to the PD-inducing neurotoxin 1-methyl-4-phenylpyridinium (MPP+). Zebrafish with parkin deficiencies did not show any abnormal mitochondrial morphology, but activity of the mitochondrial respiratory chain complex I was reduced by $45 \%$. The impairment of the mitochondrial respiratory chain is also found in the tissues of PD patients with Parkin mutations. In contrast, a different study showed no significant loss in diencephalic DA neurons after parkin knockdown, although DA neurons of the morphant fish showed an increased vulnerability to stress-induced cell death [10]. This discrepancy could result from different knockdown efficiencies of MOs targeted to different sequences in the zebrafish parkin gene.

Similar to human Parkin, zebrafish parkin is transcriptionally upregulated in response to mitochondrial stress. In addition, stable overexpression of parkin in transgenic zebrafish lines protect fish from cell death induced by proteotoxic stress, suggesting a protective capacity of parkin in vivo. This implies a potential therapy for PD by a transcriptional upregulation of endogenous Parkin [10].

\section{Pink1}

Mutations in PINK1 (PTEN-induced kinase 1) are the second most common cause of autosomal-recessive earlyonset PD. PINK1 encodes a ubiquitously expressed protein with an N-terminal mitochondrial targeting motif and a conserved serinethreonine kinase domain. PINK1 seems to protect cultures of neurons against mitochondrial dysfunction and apoptosis induced by stress. In Drosophila, pink1deficient mutants had mitochondrial defects leading to degeneration of flight muscles and mild loss of DA neurons $[11,12]$. Such defects were not observed in mice with targeted null mutations in pink1 [13, 14]. In zebrafish, pinkl is expressed ubiquitously and the predicted protein has $54 \%$ amino acid sequence identity to human PINK1. An initial study reported that MO knockdown of pinkl resulted in a an approximate $40 \%$ reduction in the number of DA neurons in the vDC [15]. However, we and others have been unable to replicate such a severe phenotype $[16$, 
17]. The phenotypes reported in the earlier study may have resulted from MO-induced effects unrelated to the pinkl gene silencing. Although pinkl knockdown did not cause large alterations in the number of DA neurons in the vDC, we observed that the patterning of these neurons and their projections were perturbed. The pinkl morphants also showed impaired response to touch stimuli and reduced swimming behavior [16]. The pinkl knockdown caused mitochondrial defects such as the loss of cristae and a reduced number of mitochondria, thus affecting mitochondrial function (unpublished data). In addition, the DA neuron clusters of pink1-deficient zebrafish were more sensitive to 1-methyl-4-phenyl-1,2,3,6-tetrapyridine (MPTP) toxicity [17]. These results indicate a role for pinkl in DA neuron development and function in zebrafish.

Developmental defects in DA neurons, resulting from PINK1 mutations, may also render DA neurons more susceptible to environmental stress. A pink1 mutant zebrafish line with a nonsense mutation in exon 7 (Y431*) was found in ENU-mutagenesis libraries [18]. This mutation is predicted to result in a partial Pink1 protein with loss of its $\mathrm{C}$ terminus and part of its kinase domain. Although there were no obvious behavioral abnormalities, the larvae ( 5 days postfertilization [dpf]) of this line showed a significant decrease in the number of DA neurons and a reduction in mitochondrial complex I activity. These phenotypes are similar to those observed in parkin-deficient zebrafish. These latter observations further supported the notion that PINK1 and Parkin are in the same pathway in regulating DA neuron development and mitochondrial functions, as was previously suggested by Drosophila PD models.

\section{$\mathrm{dj}-1$}

Mutations in DJ-1 lead to rare autosomal-recessive earlyonset PD. DJ-1, a member of the ThiJ/Pfpl/DJ-1 protein family, is involved in various functions, including its role as a redox-sensitive chaperone and in mitochondria protection against oxidative stress. In different Drosophila models RNA interference-knockdown of DJ-1 led to varying degrees of degeneration of DA neurons and hypersensitivity to oxidative stress [8]. However, similar to parkin- or PINK1-null mice, DJ-1-null mice did not show any major abnormality in the number of DA neurons in the substantia nigra pars compacta and in the levels of striatal dopamine [8]. The zebrafish $\mathrm{Dj}-1$ protein shows $83 \%$ overall amino acid identity to human DJ-1 [19]. The amino acids affected by pathogenic mutations in PD patients are especially well conserved in zebrafish $\mathrm{Dj}-1$. It is expressed through embryogenesis and transcripts are ubiquitously found in all adult tissues with a relatively higher abundance in the brain [20]. MO knockdown of $d j-1$ did not cause a decrease in number of DA neurons. However, DA neurons in $d j-1$ morphants were more sensitive to hydrogen peroxide or to the proteasome inhibitor MG132. They were also more susceptible to programmed cell death [20, 21]. Upregulation of $d j-1$ was reported in the brain of zebrafish subjected to oxidative stress [21]. These findings suggest that DJ-1 has conserved functions in zebrafish and humans. Mutations in DJ-1 may impair the response of DA neurons to environmental stress and eventually lead to cell death.

lrrk2

Mutations in $L R R K 2$ are the most frequent cause of autosomal-dominant PD. The $L R R K 2$ gene encodes a large protein with multiple functional domains, including ankyrin repeats, one Ser/Thr kinase, and one Roc GTPase enzymatic domain, as well as COR and WD40 domains. In Drosophila, overexpression of LRRK2 led to agedependent locomotor dysfunctions and loss of DA neurons $[22,23]$. The mouse transgenic models of LRRK2 (either WT or mutants) did not show any loss of DA neurons [24, 25]. Zebrafish and human LRRK2 have highly conserved amino acid sequences and predicted structures [26]. The zebrafish gene is ubiquitously expressed during embryogenesis with more abundance in the brain, and transcripts are found in multiple tissues and organs of adult fish. The deletion of the WD40 domain of 1rrk2 by splice-blocking MOs caused a significant loss of DA neurons in the vDC and locomotor defects. This also resulted in reduced and disorganized axon tracts, mainly in the midbrain. These results implicate the WD40 domain of LRRK2 in DA neuron development consistent with its known functions in mediating protein-protein interactions and regulation of LRRK2 kinase activity. Future studies should investigate the effects of overexpressing lrrk2 (WT or mutant forms) in transgenic zebrafish.

Zebrafish orthologs of other PD-related genes such as $U C H-L 1$ and GIGYF2 have also been found [27, 28]. Although several members of the synuclein family have been found in the zebrafish genome, the zebrafish ortholog of $\alpha$ synuclein has yet to be found, suggesting that the $\alpha$-synuclein gene may have been lost in zebrafish perhaps due to the functional redundancy among synuclein family members.

\section{MPTP-Induced Zebrafish PD Model}

The majority of PD cases are sporadic forms, which may suggest a role of environmental factors or more complex gene-environment interactions. The neurotoxin MPTP, known to induce PD in humans, has been successfully used in rodent and primate models of PD. It is thought that MPTP can interrupt the electron transport chain in mitochondria at complex I, which will eventually result in cell death. In adult zebrafish, MPTP induced a transient 
decrease in dopamine levels as well as behavioral defects [29]. Earlier studies in larval zebrafish showed a significant reduction of DA neurons in the vDC following MPTP treatment [30-32]. More extensive studies suggested that different groups of DA neurons in the vDC show different sensitivities to MPTP, with those neurons with ascending projections being more sensitive [33, 34]. Dopamine levels also declined after MPTP treatment, which is consistent with findings in humans. Thus, similar to mammals, MPTP can induce a significantly functional deficit of DA neurons in zebrafish.

Together, these studies strongly indicated that the DA neurons in the vDC of zebrafish are analogous to those in the mammalian nigrostriatal pathway. Zebrafish orthologs of PDrelated genes have highly conserved functions in the development and survival of DA neurons and in motor behavior. Toxininduced loss of DA neurons in zebrafish is also a relevant model of human PD. In the future, more PD-related mutants will likely be obtained using methods such as TILLING (targeting induced local lesions in genomes) or ZFN (zinc finger nucleases). These mutants might greatly facilitate the screening for small molecules with therapeutic effects.

\section{Huntington's Disease}

Huntington's disease (HD), an invariably fatal neurodegenerative disorder, follows an autosomal-dominant inheritance pattern of a mutant form of the huntingtin gene (HTT) coding for an abnormal expansion of a trinucleotide repeat encoding glutamine (CAG) at the amino terminal of the HTT protein. The length of the polyglutamine tract correlates with the penetrance, age of onset, and severity of HD. At the moment, the normal function of the HTT gene is not well understood. Despite the ubiquitous expression of $H T T$, specific brain areas of HD patients are affected earlier than others. The neuronal atrophy characteristics of HD are seen in regions of the striatum (caudate and putamen) and cortex. Medium spiny neurons of the striatum, which use $\gamma$-aminobutyric acid and project to the substantia nigra and globus pallidus, are the most vulnerable to loss [35]. What puts these neurons at risk is largely unknown.

\section{Zebrafish as a Model for HD}

The zebrafish Htt protein shares about $70 \%$ identity with the human HTT ortholog and encodes four glutamines (vs 35 glutamines in the normal human HTT) [36]. Zebrafish could prove to be a useful model, as the early embryonic death of mice with a targeted null mutation in Htt provide very limited insight into the role of HTT [37]. Zebrafish antisense $\mathrm{MO}$ knockdown, which provides a deficiency but not a total depletion of the $\mathrm{Htt}$ protein, might be a better strategy. Observations made in zebrafish htt morphant implicate wild-type $\mathrm{Htt}$ function in cellular iron utilization [38]. During gastrulation, the htt mRNA is expressed uniformly throughout the embryo; however, as development progresses, its expression decreases in non-neuronal tissues but remains strong in the head region. In the above study, a high concentration of MOs resulted in a large variety of developmental defects (slight growth delay, brain necrosis, lack of brain ventricle enlargement, and thinned yolk extension) possibly due to the off-target effects of the MOs [38]. At lower doses of MO, a thin yolk extension and blood hypochromia were the most common morphologic defects remaining and were thus deemed specific. The hypochromic blood was associated with deficits in hemoglobin production caused by altered iron metabolism. Making iron available to the embryo restored hemoglobin production in Htt-deficient embryos. Signs of iron deficiency including defects in iron homeostasis and energy metabolism are features of HD pathogenesis [39]. Thus, polyglutamine tract expansion in the wild-type huntingtin protein may alter its normal function in the metabolism of iron leading to $\mathrm{HD}$ pathology. Results from this study highlight the fact that MOs can be used successfully to produce specific phenotypes when attention is paid to the dosage.

Using the same Htt-deficient zebrafish model, Henshall et al. [40] focused on CNS defects to explain the specificity of neuropathology in HD brains. Although $h t t$ is ubiquitously expressed in the zebrafish brain, it seems to have a specific function within the forebrain that enables formation of telencephalic progenitor cells and preplacodal cells. Because the zebrafish telencephalon is believed to house the structures analogous to the human striatum, this study may have found a way of implicating a striatal-specific loss of medium-sized spiny neurons to the expression of $\mathrm{Htt}$ protein. Zebrafish deficient in Htt lose placode-derived tissue including olfactory and lateral line sensory neurons and have a reduction in telencephalic tissue. The finding that sensory neurons are perturbed by the reduction of $\mathrm{Htt}$ in the zebrafish model is consistent with the clinical observation that HD patients present with impaired olfactory function [41]. Therefore, it may be the loss of normal htt function that contributes to the symptoms of HD pathology, and not exclusively the toxic gain-of-function caused by an expansion of the polyglutamine tract.

Another in vivo study investigating $\mathrm{Htt}$ loss-of-function in zebrafish observed massive apoptosis of neuronal cells by $24 \mathrm{hpf}$, which was accompanied by impaired neuronal development, small eyes, and heads, as well as an enlargement of brain ventricles [42]. Later in development, these Htt-deficient zebrafish develop lower jaw abnormalities with most branchial arches missing. Most notably, brain-derived neurotrophic factor (BDNF) expression was 
reduced. BDNF enhances the differentiation of sensory and sympathetic neurons. The observation that Htt-MO and BDNF-MO produce similar phenotypes suggests that $\mathrm{Htt}$ regulates BDNF function. Moreover, treatment of $\mathrm{Htt}-$ deficient embryos with exogenous BDNF significantly rescued these defects. Thus, increasing expression of the prosurvival neurotrophin, BDNF, could be a therapeutic approach in the treatment of HD.

\section{Inhibition of Polyglutamine Protein Aggregation}

Schiffer et al. [43] exploited the aqueous environment of the zebrafish and the lack of its blood-brain barrier in early development to facilitate the screening of compounds that may lessen the toxicity of mutant $\mathrm{Htt}(\mathrm{mHtt})$ aggregates. As opposed to cell culture experiments, zebrafish allow for the evaluation of drugs at the whole organism level, making it possible to assess potential side effects. In HD patients, long polyglutamine tracts are found in inclusions within brain tissue. The intracellular deposition of these inclusions is associated primarily with the degeneration of neurons in the striatum. In a zebrafish HD model transiently expressing 102 polyglutamine repeats in the $\mathrm{N}$-terminal fragment of the huntingtin protein fused with GFP (Q102-GFP), this mutant protein accumulates in inclusion body-like aggregates throughout the body of embryos at $24 \mathrm{hpf}$. The soluble Q102-GFP protein translocated from the cytoplasm to inclusion bodies. Q102-GFP expression resulted in an increase in embryonic lethality or in embryos with abnormal morphology. Embryonic lethality was shown to be accompanied by apoptosis. Upon closer examination, it was seen that almost all apoptotic cells lacked inclusions. Likewise, almost all cells containing inclusions were nonapoptotic. Thus, it seems that soluble $\mathrm{mHtt}$ forms are neurotoxic, whereas insoluble inclusions containing $\mathrm{mHtt}$ aggregates are not. It was also shown that aberrantly folded Q102-GFP aggregates can be cleared by heat-shock proteins and lead to the reduction of apoptotic cells and embryonic death. This HD model's amenability to testing compounds that could inhibit Q102-GFP aggregation in vivo retrieved four inhibitors of Q102-GFP aggregation (PGL-135, Congo red, 293G02, and 306H03).

A separate study generated a stable transgenic zebrafish line expressing a Q71-GFP fusion protein under the control of the rhodopsin promoter, which was used to screen and identify compounds that reduce aggregates [44]. Thus, zebrafish have proven to be a valuable model system for the screening and identification of putative therapeutic compounds.

\section{Rhes}

Perhaps the key to the specific neuronal atrophy of striatal neurons in HD is the identification of a factor that is exclusive to these affected neurons. It has been suggested that the cytotoxic targeting of $\mathrm{mHtt}$ to striatal neurons is amplified by interactions with a mainly striatal protein called Rhes [45•]. Cell culture experiments have shown that Rhes induces sumoylation of $\mathrm{mHtt}$, which increases the amount of soluble mHTT, thus reducing $\mathrm{mHtt}$ aggregation. It has been found that disaggregated mHTT protein clumps are more neurotoxic than the mHTT aggregates [45 ${ }^{\circ}$. In an HD transgenic mouse model expressing mHTT [46], Rhes preferentially binds the striatal mHTT over the wild-type HTT protein [45•]. Cell culture studies using HEK293 cells, striatal cells, and differentiated neuronal PC12 cells reveal that the presence of Rhes reduces cell survival when mHTT is present. This mHTT-mediated reduction in cell survival was rescued by blocking Rhes with RNA interference.

Because HD diagnosis can precede the onset of symptoms, the search for drugs that block the binding of Rhes to mHTT or that block Rhes clumps from disaggregating is worthwhile and can be achieved using zebrafish to perform high-throughput small molecule screens. At the moment, more studies should be done to investigate unique features of the affected brain areas to move toward the root cause of why specific neurons are affected despite mHTT being expressed in all cells of the body.

Two possible orthologs of human Rhes in zebrafish have been annotated by Ensembl (Zv9): A4IGH1_DANRE on chromosome 1 and $z g c: 114118$ on chromosome 3 . Research into the functional characterization of the two proteins would be useful to determine if the interaction between zebrafish Rhes and mHTT is conserved. A transgenic zebrafish line expressing human mHTT-GFP or an equivalent zebrafish mHTT-GFP gene in which to examine the CNS effects of the knockdown of zebrafish Rhes may prove insightful. Questions that remain to be answered are: Following Rhes knockdown in zebrafish, would mHTT disaggregation decrease and if so, would the reduction in mHTT disaggregation correlate with a reduction in striatal neuron loss? Can compounds be identified that favor the aggregated form of mHTT over the soluble cytotoxic form in zebrafish?

Current treatments focus on reducing the symptoms of $\mathrm{HD}$, but no drug has been shown to slow the progression of the disease. The field would benefit from transgenic zebrafish models expressing the mutant polyglutamine ( $>35$ CAG repeats) form of the human HTT protein fused to GFP in a zebrafish $h t t$-null mutant background. Such a mutation could be obtained by screening for a nonsense mutation in the zebrafish $h t t$ gene by TILLING. This model would facilitate the study of the toxicity of human mHTT in its soluble versus aggregated form. Moreover, labeling of striatal medium spiny neurons in these fish would allow for performance of high-throughput screens for agents that would stop or slow the atrophy of the striatal medium spiny 
neurons. A comparison of this model to htt-deficient zebrafish models obtained by MO knockdown could be done.

\section{Alzheimer's Disease}

The pathologic hallmarks of Alzheimer's disease (AD) are extracellular amyloid- $\beta$ (A $\beta)$ protein-containing neuritic plaques and intracellular hyperphosphorylated tau-containing neurofibrillary tangles. Early-onset $\mathrm{AD}$ is associated with mutations in three genes involved in $\mathrm{A} \beta$ proteolysis displaying autosomal-dominant inheritance patterns in humans: amyloid- $\beta$ precursor protein $(A P P)$, Presenilin 1 (PSEN1), and Presenilin 2 (PSEN2). Late-onset $\mathrm{AD}$ is linked to a number of genetic risk factors including the apolipoprotein $\mathrm{E}$ (ApoE), which is a cholesterol transport protein, and the neuronal sortilin-related receptor (SORL1), which acts as a sorting receptor for APP.

In humans, APP undergoes post-translational processing. If APP is cleaved by $\alpha$-secretase, a benign A $\beta$ peptide is produced. Alternatively, APP can undergo two sequential cleavages by $\beta$-secretase and $\gamma$-secretase to generate the pathogenic $A \beta$ peptide pushing it toward the late endosomal pathway. Depending on where the $\gamma$-secretase cleaves the $A \beta C$-terminal, either $A \beta 42$ or $A \beta 40$ will be generated. $A \beta 42$ is the longer form of $A \beta$, which is more cytotoxic than the shorter $A \beta 40$ peptide.

According to the amyloid hypothesis, deposits of $A \beta$ outside the neuron are the underlying cause of AD. The competing tau hypothesis states that the hyperphosphorylated tau protein, which forms neurofibrillary tangles inside neurons, is the catalyst for $\mathrm{AD}$ disease progression. This leads to the disassembly of microtubules essential for neuronal transport, disrupting neurotransmitter communication between neurons, and ultimately resulting in cell death.

\section{Zebrafish as a Model for AD}

Several of the human genes encoding the enzymes required for the post-translational modifications of APP have been found with a high percent of amino acid similarity in zebrafish. Zebrafish have two genes similar to human APP: appa and appb [47]. During gastrulation, both app genes are expressed in the entire embryo, whereas at $24 \mathrm{hpf}$, the appa and appb paralogs are expressed in the telencephalon, the $\mathrm{vDC}$, the trigeminal ganglia, and the posterior lateral line ganglia. Orthologs of the $\beta$-secretase and $\gamma$-secretase complexes are found in zebrafish and are expressed in the CNS $[48,49]$. Despite conservation of the $\mathrm{A} \beta$ domain and of the secretases between zebrafish and humans, a zebrafish $\mathrm{A} \beta$ peptide has yet to be found and it is not known if the above post-translational modifications that occur in human APP processing also occur in zebrafish.
Loss of zebrafish Appa and Appb function by MO knockdown resulted in reduced body length and defective convergent-extension movements during gastrulation [50]. Interestingly, these defects are rescued by wild-type human APP mRNA, but not by the Swedish mutant APP, known to cause familial AD. Both zebrafish psen 1 and psen 2 are expressed ubiquitously during embryogenesis; however, psen 2 is more restricted to the CNS, eye, and spinal cord at $1 \mathrm{dpf}[48,49]$. One ortholog of the human $\beta$-secretase enzyme has been annotated by Ensembl (Zv9) in zebrafish: BACE1 on chromosome 15. Further characterization of this gene in zebrafish is required to determine if it functions similarly in zebrafish as it does in humans. Another candidate gene that requires more in-depth characterization is the ApoE $\varepsilon 4$ susceptibility gene, whose function in zebrafish is not well understood. ApoE is expressed in the zebrafish eyes, and some cells of the mesencephalic, telencephalic, and rhombencephalic brain areas, suggesting that it may play a significant function in the CNS [51].

\section{Tau}

To understand how the microtubule-associated protein tau (MAPT) contributes to tau pathology and how tau redistributes from neuronal axons to neuronal soma forming pathogenic neurofibrillary tangles, a zebrafish model transiently expressing mutant human tau has been reported [52]. In this study, human tau carrying mutations at sites associated with hereditary dementias was fused to GFP while under the control of the zebrafish pan-neural-specific GATA-2 promoter. GFP-positive neurons were found in the brain, retina, and spinal cord. In the brain, there was evidence of hallmark AD-associated cytoskeletal pathology, including disruption of tau trafficking and cytoskeletal filaments in the axon, accumulation of tau in the cell body near the axon, accumulation of fibrillar tau throughout the cell body, and presence of hyperphosphorylated tau. Despite showing that mutant human tau-GFP is hyperphosphorylated and could be used to monitor the formation of tangles, this strategy failed to generate a transgenic line stably expressing tau-GFP.

Bai et al. [53] established a stable transgenic zebrafish line expressing either GFP or mutant human 4-repeat tau under the control of the zebrafish enolase-2 promoter, $\mathrm{Tg}$ (eno2:GFP) or $\operatorname{Tg}($ eno2:Tau), respectively. Zebrafish enolase-2 was chosen because its expression in differentiated neuronal axons starts after the early stages of zebrafish development. This prevents pathogenic tau from disrupting the development of neuronal precursors and from causing defects that are not attributable to late-onset neurodegeneration. In $\operatorname{Tg}($ eno2:Tau $)$ zebrafish, tau was expressed throughout the CNS and accumulations were present in the retina, spine, axons, and neuronal soma throughout the 
brain. Stable expression of tau into adulthood will facilitate the examination of pathologic tau in the age-related progression of $\mathrm{AD}$.

Stable transgenic zebrafish expressing human tau with a mutation (TAU-P301L) found in frontotemporal dementia has been generated [54••]. TAU-P301L is expressed in zebrafish neurons using the Gal4-UAS system, where DsRed and TAU-P301L are expressed concomitantly in early neuronal cells (under the control of the zebrafish $\mathrm{HuC}$ promoter), permitting the observation of red tau-expressing cell in embryos. TAU-P301L expression showed pathologic features of tauopathies, including human tau hyperphosphorylation, tangle formation, and neurodegeneration in the spinal cord. Moreover, tau-expressing embryos have behavioral deficits in escape response after touch stimulus. This study also reported testing of inhibitors of the tau kinase GSK $3 \beta$, which lead to reduced tau phosphorylation in vivo. Thus, this model serves as a valuable tool to study $\mathrm{AD}$ and related tauopathies in vivo, especially because tau hyperphosphorylation developed within only $32 \mathrm{~h}$.

\section{SORL1}

It has been shown that APP can be diverted away from the late endosomal pathway by a SORL1-dependent switch, which sequesters APP into recycling endosomes, preventing the formation of $A \beta\left[55^{\circ}\right]$. Reduced expression of SORL1 is seen in AD brain tissue and is associated with an increase in $A \beta$ production [56]. This genetic association between AD and SORL1 expression is a result of single nucleotide polymorphisms (SNPs) found within the SORL1 gene [55•]. It has been demonstrated that SORL1 binds directly to APP and differentially regulates the sorting of APP into the late endosomal pathway leading to the production of cytotoxic $A \beta$ or into the retromer recycling pathway, sequestering APP from the $\beta$ - and $\gamma$-secretases. The overexpression of SORL1 in HEK cells reduces $A \beta$ production by $82 \%$, likely by diverting APP into the retromer recycling pathway. Controversy does exist over whether or not there is an association between AD and SORL1. Currently, more research into the link between SORL1 and late-onset AD risk is necessary. Of potential benefit in the elucidation of a link between SORL1 and $\mathrm{AD}$, a gene coding for a Sorl1-like protein is located on zebrafish chromosome 15, according to the latest Ensembl version of the zebrafish genome (Zv9). Expression and function of zebrafish sorll have yet to be investigated.

\section{Conclusions}

Animal models have proven essential for our understanding of the biological fundamentals of complex diseases. Here, we reviewed recent progress on the pathologic study of human neurodegenerative diseases using zebrafish as a model. Gene knockdown of zebrafish orthologs or transgenic expression of pathogenic genes implicated in human neurodegenerative diseases arouses the key morphologic, physiologic, and biochemical abnormalities on specific neuron groups, which are shared with other animal models and human patients. This suggested a significant level of functional conservation between the genes related to human neurodegenerative diseases and their zebrafish orthologs. Thus, zebrafish could be a good alternative model in elucidating the molecular basis of human neurodegenerative diseases. Taking advantage of some unique features of the zebrafish, we can anticipate its increased adoption as a vertebrate model for high-throughput drug screening.

Acknowledgment Research in M. Ekker's laboratory is supported by the Canadian Institutes of Health Research.

Disclosure No potential conflicts of interest relevant to this article were reported.

Open Access This article is distributed under the terms of the Creative Commons Attribution Noncommercial License which permits any noncommercial use, distribution, and reproduction in any medium, provided the original author(s) and source are credited.

\section{References}

Papers of particular interest, published recently, have been highlighted as:

- Of importance

- Of major importance

1. •- Eisen JS, Smith JC. Controlling morpholino experiments: don't stop making antisense. Development. 2008;135:1735-43. This article comprehensively described how to properly design and interpret $M O$ experiments in zebrafish.

2. Thermes V, Grabher C, Ristoratore F, et al. I-SceI meganuclease mediates highly efficient transgenesis in fish. Mech Dev. 2002;118:91-8.

3. Kawakami K, Shima A, Kawakami N. Identification of a functional transposase of the Tol2 element, an Ac-like element from the Japanese medaka fish, and its transposition in the zebrafish germ lineage. Proc Natl Acad Sci USA. 2000;97:11403-8.

4. Driever W, Solnica-Krezel L, Schier AF, et al. A genetic screen for mutations affecting embryogenesis in zebrafish. Development. 1996;123:37-46.

5. Rink E, Wullimann MF. Connections of the ventral telencephalon and tyrosine hydroxylase distribution in the zebrafish brain (Danio rerio) lead to identification of an ascending dopaminergic system in a teleost. Brain Res Bull. 2002;57:385-7.

6. Greene JC, Whitworth AJ, Kuo I, et al. Mitochondrial pathology and apoptotic muscle degeneration in Drosophila parkin mutants. Proc Natl Acad Sci USA. 2003;100:4078-83.

7. Whitworth AJ, Theodore DA, Greene JC, et al. Increased glutathione S-transferase activity rescues dopaminergic neuron 
loss in a Drosophila model of Parkinson's disease. Proc Natl Acad Sci USA. 2005;102:8024-9.

8. Dawson TM, Ko HS, Dawson VL. Genetic animal models of Parkinson's disease. Neuron. 2010;66:646-61.

9. Flinn L, Mortiboys H, Volkmann K, et al. Complex I deficiency and dopaminergic neuronal cell loss in parkin-deficient zebrafish (Danio rerio). Brain. 2009;132(Pt 6):1613-23.

10. Fett ME, Pilsl A, Paquet D, et al. Parkin is protective against proteotoxic stress in a transgenic zebrafish model. PLoS One. 2010; 5:doi:10.1371/journal.pone.0011783.

11. Clark IE, Dodson MW, Jiang C, et al. Drosophila pink1 is required for mitochondrial function and interacts genetically with parkin. Nature. 2006;441:1162-6.

12. Park J, Lee SB, Lee S, et al. Mitochondrial dysfunction in Drosophila PINK1 mutants is complemented by parkin. Nature. 2006;441:1157-61.

13. Kitada T, Pisani A, Porter DR, et al. Impaired dopamine release and synaptic plasticity in the striatum of PINK1-deficient mice. Proc Natl Acad Sci USA. 2007;104:11441-6.

14. Gautier CA, Kitada T, Shen J. Loss of PINK1 causes mitochondrial functional defects and increased sensitivity to oxidative stress. Proc Natl Acad Sci USA. 2008;105:11364-9.

15. Anichtchik O, Diekmann H, Fleming A, et al. Loss of PINK1 function affects development and results in neurodegeneration in zebrafish. J Neurosci. 2008;28:8199-207.

16. Xi Y, Ryan J, Noble $\mathrm{S}$, et al. Impaired dopaminergic neuron development and locomotor function in zebrafish with loss of pink1 function. Eur J Neurosci. 2010;31:623-33.

17. Sallinen V, Kolehmainen J, Priyadarshini M, et al. Dopaminergic cell damage and vulnerability to MPTP in Pink1 knockdown zebrafish. Neurobiol Dis. 2010;40:93-101.

18. Bandmann O, Flinn L, Mortiboys H. POMD08 Zebrafish models for early onset Parkinson's disease. J Neurol Neurosurg Psychiatry. 2010;81: doi:10.1136/jnnp.2010.226340.168.

19. Bai Q, Mullett SJ, Garver JA, et al. Zebrafish DJ-1 is evolutionarily conserved and expressed in dopaminergic neurons. Brain Res. 2006;1113:33-44.

20. Bretaud $\mathrm{S}$, Allen $\mathrm{C}$, Ingham PW, et al. p53-dependent neuronal cell death in a DJ-1-deficient zebrafish model of Parkinson's disease. J Neurochem. 2007;100:1626-35.

21. Baulac S, Lu H, Strahle J, et al. Increased DJ-1 expression under oxidative stress and in Alzheimer's disease brains. Mol Neurodegener. 2009;4:12.

22. Liu Z, Wang X, Yu Y, et al. A Drosophila model for LRRK2linked parkinsonism. Proc Natl Acad Sci USA. 2008;105:2693-8.

23. Ng CH, Mok SZ, Koh C, et al. Parkin protects against LRRK2 G2019S mutant-induced dopaminergic neurodegeneration in Drosophila. J Neurosci. 2009;29:11257-62.

24. Li Y, Liu W, Oo TF, et al. Mutant LRRK2(R1441G) BAC transgenic mice recapitulate cardinal features of Parkinson's disease. Nat Neurosci. 2009;12:826-8.

25. Li X, Patel JC, Wang J, et al. Enhanced striatal dopamine transmission and motor performance with LRRK2 overexpression in mice is eliminated by familial Parkinson's disease mutation G2019S. J Neurosci. 2010;30:1788-97.

26. Sheng D, Qu D, Kwok KH, et al. Deletion of the WD40 domain of LRRK2 in Zebrafish causes Parkinsonism-like loss of neurons and locomotive defect. PLoS Genet. 2010;6:doi:10.1371/journal. pgen.1000914.

27. Son OL, Kim HT, Ji MH, et al. Cloning and expression analysis of a Parkinson's disease gene, uch-L1, and its promoter in zebrafish. Biochem Biophys Res Commun. 2003;312:601-7.

28. Guella I, Pistocchi A, Asselta R, et al. Mutational screening and zebrafish functional analysis of GIGYF2 as a Parkinson-disease gene. Neurobiol Aging. 2010;doi:10.1016/j.neurobiolaging. 2009.12.016
29. Anichtchik OV, Kaslin J, Peitsaro N, et al. Neurochemical and behavioural changes in zebrafish Danio rerio after systemic administration of 6-hydroxydopamine and 1-methyl-4-phenyl-1, 2, 3, 6-tetrahydropyridine. J Neurochem. 2004;88:443-53.

30. Lam CS, Korzh V, Strahle U. Zebrafish embryos are susceptible to the dopaminergic neurotoxin MPTP. Eur J Neurosci. 2005;21:1758-62.

31. Bretaud S, Lee S, Guo S. Sensitivity of zebrafish to environmental toxins implicated in Parkinson's disease. Neurotoxicol Teratol. 2004;26:857-64.

32. McKinley ET, Baranowski TC, Blavo DO, et al. Neuroprotection of MPTP-induced toxicity in zebrafish dopaminergic neurons. Brain Res Mol Brain Res. 2005;141:128-37.

33. Wen $\mathrm{L}$, Wei $\mathrm{W}, \mathrm{Gu} \mathrm{W}$, et al. Visualization of monoaminergic neurons and neurotoxicity of MPTP in live transgenic zebrafish. Dev Biol. 2008;314:84-92.

34. Sallinen V, Torkko V, Sundvik M, et al. MPTP and MPP+ target specific aminergic cell populations in larval zebrafish. J Neurochem. 2009;108:719-31.

35. Graveland GA, Williams RS, DiFiglia M. Evidence for degenerative and regenerative changes in neostriatal spiny neurons in Huntington's disease. Science. 1985;227:770-3.

36. Karlovich CA, John RM, Ramirez L, et al. Characterization of the Huntington's disease (HD) gene homologue in the zebrafish Danio rerio. Gene. 1998;217:117-25.

37. Zeitlin S, Liu JP, Chapman DL, et al. Increased apoptosis and early embryonic lethality in mice nullizygous for the Huntington's disease gene homologue. Nat Genet. 1995;11:155-63.

38. Lumsden AL, Henshall TL, Dayan S, et al. Huntingtin-deficient zebrafish exhibit defects in iron utilization and development. Hum Mol Genet. 2007;16:1905-20.

39. Morrison PJ, Nevin NC. Serum iron, total iron binding capacity and ferritin in early Huntington disease patients. Ir J Med Sci. 1994;163:236-7.

40. Henshall TL, Tucker B, Lumsden AL, et al. Selective neuronal requirement for huntingtin in the developing zebrafish. Hum Mol Genet. 2009; 18:4830-42.

41. Pirogovsky E, Gilbert PE, Jacobson M, et al. Impairments in source memory for olfactory and visual stimuli in preclinical and clinical stages of Huntington's disease. J Clin Exp Neuropsychol. 2007;29:395-404.

42. Diekmann H, Anichtchik O, Fleming A, et al. Decreased BDNF levels are a major contributor to the embryonic phenotype of huntingtin knockdown zebrafish. J Neurosci. 2009;29:1343-9.

43. Schiffer NW, Broadley SA, Hirschberger T, et al. Identification of anti-prion compounds as efficient inhibitors of polyglutamine protein aggregation in a zebrafish model. J Biol Chem. 2007;282:9195-203.

44. Williams A, Sarkar S, Cuddon P, et al. Novel targets for Huntington's disease in an mTOR-independent autophagy pathway. Nat Chem Biol. 2008;4:295-305.

45. - Subramaniam S, Sixt KM, Barrow R, et al. Rhes, a striatal specific protein, mediates mutant-huntingtin cytotoxicity. Science. 2009;324:1327-30. The Rhes protein found specifically in the mammalian striatum may explain the specific death of medium spiny neurons in $H D$.

46. Schilling G, Becher MW, Sharp AH, et al. Intranuclear inclusions and neuritic aggregates in transgenic mice expressing a mutant $\mathrm{N}$ terminal fragment of huntingtin. Hum Mol Genet. 1999;8:397-407.

47. Musa A, Lehrach H, Russo VA. Distinct expression patterns of two zebrafish homologues of the human APP gene during embryonic development. Dev Genes Evol. 2001;211:563-7.

48. Leimer U, Lun K, Romig $\mathrm{H}$, et al. Zebrafish (Danio rerio) presenilin promotes aberrant amyloid beta-peptide production and requires a critical aspartate residue for its function in amyloidogenesis. Biochemistry. 1999;38:13602-9. 
49. Groth C, Nornes S, McCarty R, et al. Identification of a second presenilin gene in zebrafish with similarity to the human Alzheimer's disease gene presenilin2. Dev Genes Evol. 2002;212:486-90.

50. Joshi P, Liang JO, DiMonte K, et al. Amyloid precursor protein is required for convergent-extension movements during Zebrafish development. Dev Biol. 2009;335:1-11.

51. Babin PJ, Thisse C, Durliat M, et al. Both apolipoprotein E and AI genes are present in a nonmammalian vertebrate and are highly expressed during embryonic development. Proc Natl Acad Sci USA. 1997;94:8622-7.

52. Tomasiewicz HG, Flaherty DB, Soria JP, et al. Transgenic zebrafish model of neurodegeneration. J Neurosci Res. 2002;70:734-45.

53. Bai Q, Garver JA, Hukriede NA, et al. Generation of a transgenic zebrafish model of Tauopathy using a novel promoter element derived from the zebrafish eno2 gene. Nucleic Acids Res. 2007;35:6501-16.
54. •- Paquet D, Bhat R, Sydow A, et al. A zebrafish model of tauopathy allows in vivo imaging of neuronal cell death and drug evaluation. J Clin Invest. 2009;119:1382-95. Generation of a stable transgenic Gal4-UAS-based zebrafish model expressing human tau with a mutation found in frontotemporal dementia AD patients shows pathologic features of tauopathies.

55. - Rogaeva E, Meng Y, Lee JH, et al. The neuronal sortilin-related receptor SORL1 is genetically associated with Alzheimer disease. Nat Genet. 2007;39:168-77. AD-associated SNPs are found in the SORL1 protein, which normally helps sequester APP from cleavage enzymes that generate cytotoxic $A \beta$.

56. Andersen OM, Reiche J, Schmidt V, et al. Neuronal sorting protein-related receptor sorLA/LR11 regulates processing of the amyloid precursor protein. Proc Natl Acad Sci USA. 2005;102:13461-6. 